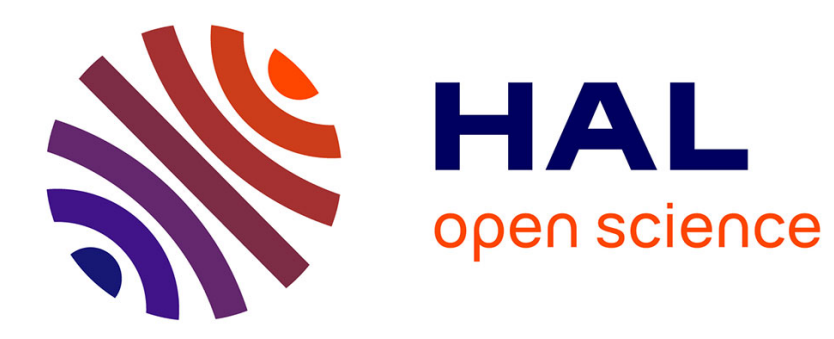

\title{
Sur Un Probleme Combinatoire D'Antennes En Radioastronomie II
}

Jean-Claude Bermond, G. Farhi

\section{To cite this version:}

Jean-Claude Bermond, G. Farhi. Sur Un Probleme Combinatoire D'Antennes En Radioastronomie II. Annals of Discrete Mathematics, 1982, 12, pp.49-53. 10.1016/S0304-0208(08)73490-X . hal-02424286

\section{HAL Id: hal-02424286 \\ https://hal.inria.fr/hal-02424286}

Submitted on 26 Dec 2019

HAL is a multi-disciplinary open access archive for the deposit and dissemination of scientific research documents, whether they are published or not. The documents may come from teaching and research institutions in France or abroad, or from public or private research centers.
L'archive ouverte pluridisciplinaire HAL, est destinée au dépôt et à la diffusion de documents scientifiques de niveau recherche, publiés ou non, émanant des établissements d'enseignement et de recherche français ou étrangers, des laboratoires publics ou privés. 


\section{SUR UN PROBLEME COMBINATOIRE D'ANTENNES EN RADIOASTRONOMIE II}

\section{J. C. BERMOND et G. FARHI}

Laboratoire de Recherche en Informatique, E.R.A. 452-Bâtiment 490, Université de Paris-Sud-91405 Orsay, France

\section{Dédié au Professeur A. Kotzig à l'occasion de son soixantième anniversaire}

Dans cet article, nous donnons un théorème de non existence pour des systèmes généralisés de différences intervenant dans un problème d'antennes en radioastronomie. Le théorème peut s'exprimer sous la forme suivante: le graphe formé de $m K_{n}$ ayant exactement un $K_{q}$ en commun n'est pas gracieux dès que $n$ est assez grand par rapport à $q$.

In this paper, we give a non-existence theorem concerning generalized systems of difference sets, which arise in a problem of antennas in radioastronomy. This theorem is equivalent to the fact that the graph consisting of $m K_{n}$ having exactly $a K_{q}$ in common is not graceful for $n$ sufficiently large compared to $q$

\section{Introduction}

Définition. On considère $m$ ensembles d'entiers $(m \geqslant 2), A_{i}=\left\{a_{i 1}, a_{i 2}, \ldots, a_{i n}\right)$ où les $a_{i j}$ sont rangés par ordre croissant, plus exactement: $0=a_{i 1}<a_{i 2}<\cdots<a_{i n}$.

On suppose de plus que les $A_{i}$ ont $q$ éléments en commun; soit $A=\left\{a_{j}: 1 \leqslant j \leqslant q\right\}$ l'ensemble des valeurs communes.

Soit $D_{i}=\left\{\left(a_{i j}-a_{i h}\right): 1 \leqslant h<j \leqslant n\right\}$ l'ensemble des différences associé à $A_{i}$ et soit $\Delta=\left\{\left|a_{j}-a_{h}\right|: 1 \leqslant h<j \leqslant q\right\}$ (différences entre les $q$ éléments en commun).

Posons $D=\bigcup_{i=1}^{m} D_{i}$ (notons que $D_{i} \supset \Delta, 1 \leqslant i \leqslant m$ ). On dit que les ensembles $A_{i}$ forment un $(m, n) q$-système si

$$
D=\left\{1,2, \ldots, \frac{m n(n-1)}{2}-(m-1) \frac{q(q-1)}{2}\right\}
$$

c'est-à-dire $D$ est formé d'entiers consécutifs distincts.

Exemples. $m=4, n=3, q=1$ :

$$
\begin{array}{rlrl}
A_{1} & =\{0,4,6\} & A_{2}=\{0,9,10\}, & \\
A_{3} & =\{0,8,11\}, & A_{4}=\{0,7,12\} . & \\
m=3, n=4, q=3: & & \\
A_{1}=\{0,2,5,12\}, & D_{1}=\{2,3,5,7,10,12\}, \\
A_{2}=\{0,2,8,12\}, & D_{2}=\{2,4,6,8,10,12\}, \\
A_{3}=\{0,2,11,12\}, & D_{3}=\{1,2,9,10,11,12\} .
\end{array}
$$


Remarque. Le cas $q=1$ correspond au cas classique du problème d'astronomie (antennes toutes mobiles) et est appelé dans [3] un système parfait.

Rappelons qu'un graphe $G$ ayant $e$ arêtes est dit gracieux si on peut numéroter les sommets de $G$ avec des entiers, tous distincts, de $\{0,1, \ldots, e\}$ de sorte que si on numérote une arête par la valeur absolue de la différence des numéros de ses sommets les $e$ arêtes aient pour valeurs tous les entiers de $\{1,2, \ldots, e\}$. L'existence d'un $(m, n)$ $q$-système revient à déterminer si le graphe formé de $m K_{n}$ ayant exactement un $K_{q}$ en commun est gracieux, problème posé en [5] et étudié en [9].

\section{Résultats antérieurs}

Cas $q=1$ (systèmes parfaits).

Dans [3] il est montré que si un $(m, n) 1$-système existe alors $n \leqslant 5$, avec de plus si $n=5, m$ pair $\geqslant 4$ et $\operatorname{si} n=3, m \equiv 0$ ou $1(\bmod 4)$.

Dans $[2,3]$ il est montré qu'un $(m, 3) 1$-système existe si (et seulement si) $m \equiv 0$ ou 1 $(\bmod 4)$.

Dans [3] il est montre qu'un ( $m, 4) 1$-système existe pour $m=1,4,5$, 6 et n'existe pas pour $m=2,3$. Mills (communication privée) a construit de tels systèmes pour $m=7$ et 8 .

Dans [4] il est conjecturé qu'un $(m, 4) 1$-système existe pour $m \geqslant 4$. Kotzig et Turgeon $[11]$ et Rogers $[13,14]$ ont développé des méthodes ('théorèmes d'addition') permettant de construire à partir d'un $(m, 4) 1$-système des $\left(m^{\prime}, 4\right)$ 1-système pour $m^{\prime}=5 m+1 ; 14 m+1[11,13,14] ; m^{\prime}=19 m+4,25 m+4,49 m+4, \ldots$ et d'autres familles de valeurs [13].

Laborde (communication privée) a montré qu'il n'existe pas de $(4,5) 1$-système et Laufer [8] a montré l'existence de $(m, 5) 1$-système pour $m=6$ et 8 .

D'autres résultats reliés à ce problème apparaissent dans [1].

Cas $q>1$.

L'existence de $(m, 3)$ 2-système est immédiate (voir aussi [10]). Delorme [6] a montré que pour tout $m$ il existe un $(m, 4)$ 2-système. Meyniel (communication privée, voir [5]) a établi l'existence de $(m, 4) 3$-système pour tout $m$, (voir aussi [10]).

Récemment Farhi [7] a montré qu'il n'existait pas de $(m, 5) 4$-système.

Nous montrons ici le théorème suivant:

Théorème. Soit $m \geqslant 2, r=\frac{1}{2} q(q-1)$ et $p=\left\lfloor\frac{1}{2}(n-1)\right\rfloor$. Alors il n'existe pas de $(m, n)$ q-système pour

$$
p \geqslant(1+\sqrt{1+4 r(2-\sqrt{2})}) / 2(\sqrt{2}-1)
$$

Remarque. Si $m$ est fixé (en particulier si $m$ est petit) la démonstration ci-dessous permet éventuellement d'exclure d'autres triplets de valeurs. D'autre part, vu la non existence des $(m, 5)$ 4-système [7] il est vraisemblable que les $(m, n) q$-systèmes n'existent presque jamais. 
Enfin, remarquons que bien souvent les graphes considérés sont eulériens (cas où $n$ est impair et $q$ impair, ou $n$ impair, $q$ pair et $m$ impair). On sait alors (cf. [5]) que le nombre d'arêtes doit être congru à 0 ou à 3 modulo 4 , ce qui exclut un certain nombre de $(m, n)$ q-systèmes.

\section{Démonstration du théorème}

Dans la suite nous poserons $p=\left[\frac{1}{2}(n-1)\right]$ et $r=\frac{1}{2} q(q-1)$.

(a) Supposons qu'il existe un $(m, n) q$-système. Nous utiliserons les notations de [2] à savoir:

$$
d_{i, j}^{k}=a_{i, j+k}-a_{i, j} ; \quad k=1,2, \ldots, n-1 ; \quad j=1,2, \ldots, n-k ; \quad i=1,2, \ldots, m .
$$

La proposition (1.1) de [2] s'écrit pour $k=1,2, \ldots, \mathrm{p}$

Soit

$$
\sum_{j=1}^{k} d_{i, j}^{n-k}=\sum_{j=1}^{n-k} d_{i, j}^{k}
$$

$$
\alpha=\sum_{i=1}^{m} \sum_{k=1}^{p} \sum_{j=1}^{n-k} d_{i, j}^{k}
$$

On a donc

$$
\alpha=\sum_{i=1}^{m} \sum_{k=1}^{p} \sum_{j=1}^{k} d_{i, j}^{n-k}
$$

(b) D'après (1), $\alpha$ est la somme de $P$ termes, avec $P=m \mid \sum_{k=1}^{p}(n-k)$. Parmi ces termes, soit $P-d$ le nombre de ceux qui sont distincts. Il y a donc $d$ répétitions; remarquons que seules peuvent être répétées les différences de la forme $a_{j}-a_{h}(1 \leqslant h<j \leqslant q)$. De plus une telle différence est répétée au plus $m-1$ fois, donc $0 \leqslant d \leqslant(m-1) r$.

Posons $d=(m-1) s+t$ avec $0 \leqslant s<r, 0 \leqslant t<m-1$, ou $s=r$ et $t=0$. Soit $\alpha(d)$ la valeur de $\alpha$ pour une valeur donnée de $d$ et soit

$$
G(d)=\sum_{i=1}^{P-d} i+(m-1) \sum_{i=1}^{s} i+t(s+1) .
$$

Alors $\alpha(d) \geqslant G(d)$. (Ceci est obtenu en considérant qu'il y a $P-d$ différences distinctes et que parmi les différences répétées il y en a $s$ distinctes répétées chacune au plus $m-1$ fois et la $(s+1)$-ième au plus $t$ fois.)

D'après (2) $\alpha$ est la somme de $N$ termes, avec $N=m \sum_{k=1}^{p} k$.

Tous ces termes sont inférieurs ou égaux à $M$ où

$$
M=\frac{1}{2} m n(n-1)-(m-1) r .
$$

Parmi ceux-ci $M-d^{\prime}$ termes sont distincts avec $d^{\prime}=(m-1) r-d$. On a au plus $d^{\prime}$ répétitions, chaque différence étant au plus répétée $(m-1)$ fois. On obtient d'une manière analogue à (3) la majoration suivante:

où

$$
\alpha(d) \leqslant D(d)
$$

$$
D(d)=\sum_{i=M-\left(N-d^{\prime}\right)+1}^{M} i+(m-1) \sum_{i=0}^{r-s-1}(M-i)-t(M-(r-s)+1) .
$$


(c) Si $G(d)>D(d)$ pour $0 \leqslant d \leqslant(m-1) r$ on obtient une contradiction et il n'existe pas de $(m, n) q$-système.

Nous allons montrer qu'en fait, cette condition est réalisée dès que $G((m-1) r)$ $>D((m-1) r)$. En effet soit $0 \leqslant d<(m-1) r$. D'après (3)

$$
G(d)-G(d+1)=P-d-(s+1)
$$

et d'après (4)

$$
D(d+1)-D(d)=M-N+d^{\prime}-(M-(r-s)+1)=m r-N-d-s-1 .
$$

Donc

$$
G(d)-D(d)-(G(d+1)-D(d+1))=P-N+m r-2(d+s+1) \geqslant P-N-m r
$$

car $d+s+1 \leqslant m r$. Or $P=\frac{1}{2} m p(2 n-1-p)$ et $N=\frac{1}{2} m p(p+1)$.

Donc $P-N-m r=m[p(n-p-1)-r]$ et cette quantité est positive dès que $r \leqslant p^{2} \leqslant p(n-p-1)$. Donc si $r<p^{2}, G(d)-D(d) \geqslant G(d+1)-D(d+1)$ et par suite

$$
G(d)-D(d) \geqslant G((m-1 r)-D((m-1) r) .
$$

Il ne nous reste plus qu'à vérifier que les hypothèses du théorème impliquent $r<p^{2}$ et $G((m-1) r)-D((m-1) r)>0$.

(d) Calculons donc $G((m-1) r)-D((m-1) r)$.

$$
G((m-1) r)=\sum_{i=1}^{P-(m-1) r} i+(m-1) \sum_{i=1}^{r} i, \quad D((m-1) r)=\sum_{i=M-N+1}^{M} i .
$$

- Dans le cas $n=2 p+2 ; P=\frac{3}{2} m p(p+1) ; N=\frac{1}{2} m p(p+1)$ et $M=m\left(2 p^{2}+3 p+1\right)$ et $G((m-1) r)-D((m-1) r)$ vaut

$$
\left.\frac{1}{4} m\left[m\left(p^{4}-(4 r+3) p^{2}-(4 r+2) p+2 r^{2}\right)+(4 r+2) p^{2}+(4 r+2) p-2 r^{2}\right)\right] .
$$

- Dans le cas $n=2 p+1, \quad P=\frac{1}{2} m p(3 p+1) ; \quad N=\frac{1}{2} m p(p+1)$ et $M=m p(2 p+1)$ et $G((m-1) r)-D((m-1) r)$ vaut

$$
\frac{1}{4} m\left[m\left(p^{4}-2 p^{3}-(4 r+1) p^{2}+2 r^{2}\right)+(4 r+2) p^{2}-2 r^{2}\right] .
$$

Soit $p \geqslant(1+\sqrt{1+4 r(2-\sqrt{2}})) / 2(\sqrt{2}-1)$ (ce qui implique en particulier $p \geqslant 2$ ). Alors $r \leqslant r_{0}=\left(1-\frac{1}{2} \sqrt{2}\right) p^{2}-\frac{1}{2} \sqrt{2} p$. Donc $r \leqslant p^{2}$ et par suite les coefficients de $m$ dans (5) et (6) sont strictement positifs.

Soit $A(r)$ (resp. $\left.A^{\prime}(r)\right)$ le coefficient en $m^{2}$ dans (5) (resp. (6)). Comme $r \leqslant p^{2}, A(r) \geqslant A\left(r_{0}\right)$ $=2(\sqrt{2}-1)\left(p^{3}+p^{2}\right)-2 p \geqslant 0(\operatorname{car} p \geqslant 2)$ et $A^{\prime}(r) \geqslant A^{\prime}\left(r_{0}\right)=0$.

Remarque. On peut considérer un problème plus général en supposant que $D$ est formé d'entiers consecutifs distincts $\geqslant c$ ( $c$ entier donné) (voir [2] pour le cas $q=1$ ). Rogers (communication privée) a montré que le théorème restait vrai pour de tels systèmes. On peut aussi considérer des ensembles $A_{i}$ de tailles différentes (voir [1] et [12] pour le cas $q=1$ ). 
Nous remercions particulièrement D. G. Rogers pour ses remarques qui ont permis d'améliorer l'article.

\section{Bibliographie}

[1] J. Abrham, Bounds for the sizes of components in perfect systems of difference sets, dans ce volume, pp. 1-7.

[2] J. C. Bermond, A. E. Brouwer et A. Germa, Systèmes de triplets et différences associées, dans: Coll. CNRS, Problèmes Combinatoires et Théorie des Graphes, Orsay, 1976 (CNRS, 1978) pp. 35-38.

[3] J. C. Bermond, A. Kotzig et J. Turgeon, On a combinatorial problem of antennas in radioastronomy, dans Combinatorics, Coll. Math. Soc. Janos Bolyai No. 18, Keszthely, 1976 (North-Holland Amsterdam, 1978) pp. 135-149.

[4] J. C. Bermond, Problem dans combinatorics, Coll. Math. Soc. Janós Bolyai No. 18, Keszthely, 1976 (North-Holland Amsterdam, 1978) p. 1189.

[5] J. C. Bermond, Graceful graphs, radio antenna and French windmills, dans: Proc. One Day Conference on Combinatorics Open University, 1978, Research Notes in Mathematics (Pitman London, 1979) pp. 18-37.

[6] C. Delorme, Two sets of graceful graphs, J. Graph Theory 4 (1980) 247-250.

[7] G. Farhi, Thèse, Informatique, Université Paris-Sud (1981).

[8] P. J. Laufer, Regular perfect systems of difference sets of size five, dans ce volume, pp. 193-201.

[9] K. M. Koh, D. G. Rogers, H. K. Teo et K. Y. Yap, Graceful graphs: some further results and problems. Research Report 91, College of Graduate studies, Nanyang U. Singapour (1980).

[10] K. M. Koh, D. G. Rogers et C. K. Lim, On graceful graphs: sum of graphs, Research Report 78, College of Graduate Studies, Nanyang U. Singapour (1979).

[11] A. Kotzig et J. Turgeon, Perfect systems of difference sets and additive sequences of permutations, dans: Proc. 10th Southeastern Conference of Combinatorics, Graph Theory and Computing, Boca Raton (1979), Utilitas Math. Publ. Congressus Numerantium 24, 629-636.

[12] A. Kotzig et J. Turgeon, Sur l'existence de petites composantes dans tout système parfait d'ensembles de différences, dans: Proc. Coll. Montréal, 1979, Annals of Discrete Math. 8 (North-Holland, Amsterdam, 1980) pp. 71-75.

[13] D. G. Rogers, Addition theorems for perfect systems of difference sets, J. London Math. Soc. 23 (1981) 385-395.

[14] D. G. Rogers, A multiplication theorem for perfect systems of difference sets, Discrete Mathematics, to appear. 